\title{
Search for light sterile neutrinos with the STEREO experiment
}

\author{
Helena Almazán ${ }^{1}$, Pablo del Amo Sanchez ${ }^{2}$, Laura Bernard ${ }^{3}$, Adrien Blanchet ${ }^{4}$, Aurélie Bonhomme ${ }^{4}$, Christian Buck $^{1}$, \\ Ilham El Atmani ${ }^{4}$, Jean Favier ${ }^{2}$, Julia Haser ${ }^{1}$, Victor Hélaine ${ }^{3}$, Felix Kandzia ${ }^{5}$, Serge Kox ${ }^{3}$, Jacob Lamblin ${ }^{3}$, Alain \\ Letourneau $^{4}$, a , David Lhuillier $^{4}$, Manfred Lindner $^{1}$, Luis Manzanillas ${ }^{2}$, Thomas Materna ${ }^{4}$, Alessandro Minotti ${ }^{4}$, François \\ Montanet ${ }^{3}$, Henri Pessard ${ }^{2}$, Jean-Sébastien Real ${ }^{3}$, Christian $\mathrm{Roca}^{1}$, Thomas Salagnac ${ }^{3}$, Stefan Schoppmann ${ }^{1}$, Viktoriya \\ Sergeyeva ${ }^{2}$, Torsten Soldner ${ }^{5}$, Anne Stutz ${ }^{3}$, and Stephane Zsoldos ${ }^{3}$ (STEREO collaboration)
}

${ }^{1}$ Max-Planck-Institut für Kernphysik, Saupfercheckweg 1, 69117 Heidelberg, Germany

${ }^{2}$ Univ. Grenoble Alpes, Université Savoie Mont Blanc, CNRS/IN2P3, LAPP, 74000 Annecy, France

${ }^{3}$ Univ. Grenoble Alpes, CNRS, Grenoble INP, LPSC-IN2P3, 38000 Grenoble, France

${ }^{4}$ Irfu, CEA, Université Paris-Saclay, 91191 Gif-sur-Yvette, France

${ }^{5}$ Institut Laue Langevin, CS 20156, 38042 Grenoble Cedex 9, France

\begin{abstract}
The STEREO experiment is searching for a non-standard oscillation in the propagation of antineutrinos produced by a nuclear reactor which could be the sign for the existence of a sterile state of $\mathrm{eV}$ mass and the origin of the reactor anti-neutrino anomaly. In this paper, results from 66 days of reactor turned on and 138 days of reactor turned off are reported excluding large amplitude oscillations. A special focus is put on the data analysis and studies of correlated backgrounds. In particular the origin of the correlated background measured in reactor turned off periods is discussed. This background mainly originates from neutrons produced by cosmic radiation.
\end{abstract}

\section{Introduction}

The STEREO experiment has been running since November 2016 at the high flux reactor of the Institut Laue-Langevin in the search for a non-standard oscillation in the short-range propagation of electron anti-neutrinos. If observed such an oscillation would be the signature for the existence of sterile neutrinos at the $\mathrm{eV}$ mass scale. This could explain the reactor anti-neutrino anomaly highlighted in 2011, when new spectral predictions of reactor anti-neutrino fluxes [1,2] showed a 6\% deficit in the global rate of previous experiments located at a few tens of meters from reactors [3]. The electron antineutrinos produced in the core of the reactor are detected in the segmented STEREO detector using the Inverse Beta Decay (IBD) reaction. The high enrichment of the nuclear fuel in ${ }^{235} \mathrm{U}(93 \%)$ allows for the measurement of a nearly pure ${ }^{235} \mathrm{U}$ anti-neutrino spectrum. The compactness of the core $(80 \mathrm{~cm}$ high, $40 \mathrm{~cm}$ in diameter) allows for the study of spectral distortions with distance over the full energy range of the reactor anti-neutrino spectrum if the mass splitting $\Delta m_{14}^{2}$ is around $1 \mathrm{eV}$. In the simplest model with one sterile neutrino, the survival probability of electron anti-neutrinos, at short distance where the impact of known oscillations due to $\Delta m_{12}^{2}$ and $\Delta m_{23}^{2}$ can be neglected, writes:

$$
P_{\overline{\bar{v}_{e}} \rightarrow \overline{v_{e}}}\left(L, E_{\overline{v_{e}}}\right) \simeq 1-\sin ^{2}\left(2 \theta_{e e}\right) \sin ^{2}\left(\frac{1.27 \Delta m_{14}^{2} L}{E_{\overline{v_{e}}}}\right)
$$

where $E_{\overline{v_{e}}}$ is the $\overline{v_{e}}$ energy in $\mathrm{MeV}$ (few $\mathrm{MeV}$ for a reactor anti-neutrino), $L$ is the distance between the

a e-mail: aletourneau@cea.fr anti-neutrino production and its detection in meters, $\Delta m_{14}^{2}$ and $\theta_{e e}$ are the mass squared difference in $\mathrm{eV}^{2}$ units and the mixing angle introduced by the additional sterile neutrino, respectively.

The test for the existence of a sterile neutrino with 66 days of reactor operating (STEREO phase I) was already published in [4] showing that oscillations with amplitudes greater than $15-20 \%$ could be rejected with more than $90 \%$ C.L in the mass squared difference range of $1-5 \mathrm{eV}^{2}$. In the present paper we detail the analysis method used in [4], in particular the methodology to separate the anti-neutrino events from the background, and the recent improvements resulting from a better knowledge of the background and its origin thanks to a long reactor turned off period during STEREO phase II. These improvements do not impact the results from [4] but are important to extract the absolute anti-neutrino rates and the energy spectrum associated with the fission of ${ }^{235} \mathrm{U}$ to test the shape distortions above $4-5 \mathrm{MeV}$ observed in other reactor anti-neutrino experiments (see [5] and references therein for discussions).

\section{STEREO experiment}

\subsection{Detector}

The detector consists of six identical target cells placed along the anti-neutrino trajectory covering distances between 9.4 to $11.2 \mathrm{~m}$ with respect to the fuel element (see Fig. 1). Each cell is $37 \mathrm{~cm}$ thick, $90 \mathrm{~cm}$ high and $90 \mathrm{~cm}$ large, and filled with gadolinium-loaded organic liquid scintillator allowing electron anti-neutrinos to be detected via the inverse beta decay reaction on hydrogen

(C) The Authors, published by EDP Sciences. This is an Open Access article distributed under the terms of the Creative Commons Attribution License 4.0 (http://creativecommons.org/licenses/by/4.0/). 


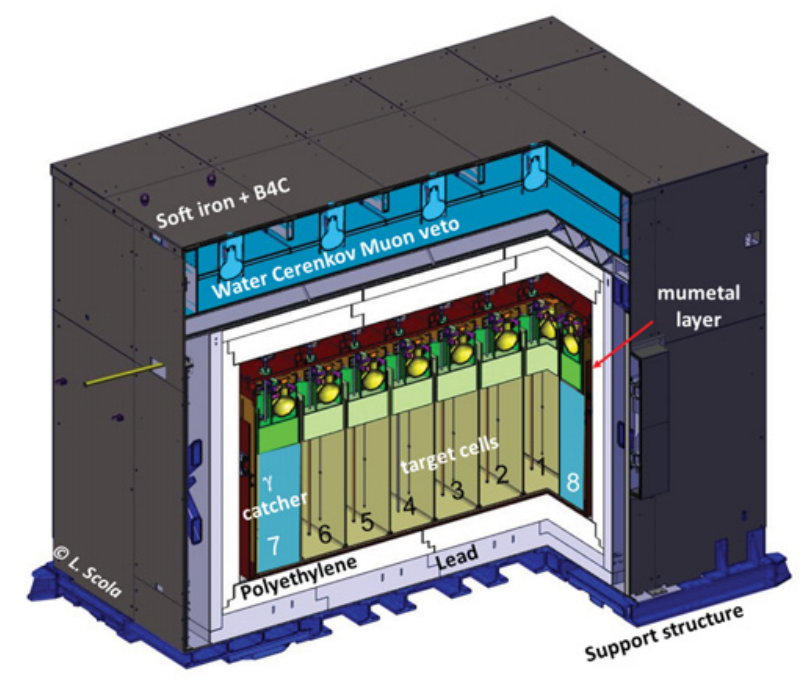

Figure 1. STEREO setup showing the target cells (1-6), two of the gamma catcher cells $(7 \& 8)$ and the shieldings.

nuclei: $\bar{v}_{e}+p \rightarrow e^{+}+n$. In this reaction two particles are produced in the final state. The signature of an IBD event is a time coincidence between a prompt signal coming from the interaction of the positron in the scintillator and a delayed signal generated by the radiative capture of the neutron mainly on gadolinium atoms thanks to their high capture cross sections. The electron anti-neutrino energy is inferred from the positron prompt deposited energy. The prompt and delayed scintillation light are collected, in each cell, by four 8inch photomultiplier tubes (PMT) placed on the top of the cell in an acrylic buffer. Mineral oil is used to ensure the optical contact. Mirror plates built from acrylic plates and reflective foils of VM2000 are used to optically separate the cells and to improve and homogenize the light collection between events at the bottom and the top of a cell. An outer crown - called gamma catcher - filled with unloaded organic liquid scintillator serves to recover the full energy deposition loss in the target due to escaping gamma-rays. More details on detector and shielding design can be found in [6].

\subsection{Background mitigation}

The location of the STEREO detector at ground level inside a reactor environment makes the measurement challenging: on top of natural radioactivity, STEREO is exposed to radiations coming from the reactor core, from the neighboring experiments, and from cosmic particles. A passive shielding is used to reduce the gamma ray and neutron background mainly coming from the neighboring environment. From outside to inside it is composed of a boron carbide layer to absorb thermal neutrons, a soft iron layer for magnetic shielding, a lead gammaray shielding, a borated polyethylene neutron moderator and absorber and a mu-metal magnetic shielding. A water Cherenkov muon veto detector is placed on the top of the STEREO setup to act as active shielding by tagging crossing muons. Topological information and Pulse Shape Discrimination (PSD) properties of the liquid scintillator, i.e., the capability to discriminate between proton recoils and electron recoils due to the different decay times of the respective scintillation light pulses, are also used to improve the background rejection. The remaining components are measured during reactor turned off periods (see Sect. 3.2).

\subsection{Light response monitoring and calibration}

The detector light response and stability are continuously monitored. A light injection system is used to calibrate the PMTs at the photo-electron level and to monitor the linearity of the electronics, PMTs and evolution of the liquids' properties. A set of radioactive gamma sources (covering the energy range from 0.5 to $4.4 \mathrm{MeV}$ ) is also regularly deployed inside and all around the detector to monitor the detector response and to calibrate it.

As the optical separations between cells are not perfect and evolving with time, especially during the STEREO phase I, we developed a method to convert the measured PMT signals into a reconstructed energy. Using this method, time-drifts of the reconstructed energy for (n, $\gamma$ ) reactions on hydrogen and gadolinium isotopes are less than $1 \%$ and the energy resolution $(\Delta E / E$ at $1 \sigma)$ for ${ }^{54} \mathrm{Mn}$ gamma rays is about $9 \%$. Energy nonlinearity due to the quenching of the liquid is measured precisely up to 4.4 MeV and reproduced by the Monte-Carlo simulation of the detector, based on Geant4 libraries, at the percent level. The cell-to-cell deviation between the data and the MonteCarlo is also controlled at the percent level. More details on the detector light response, calibration and energy reconstruction can be found in [6].

\section{Correlated events}

\subsection{IBD selection}

IBD candidates are selected by looking for coincidences between a promp event (reconstructed energy between 1.625 and $7.125 \mathrm{MeV}$ ) and a delayed event (reconstructed energy between 4.5 and $10 \mathrm{MeV}$ ) within a time window of $70 \mu \mathrm{s}$. The contribution of random coincidences of two uncorrelated signals in the coincidence time window is measured during the pair selection process by looking for delayed events in many delayed time windows for each prompt event (typically 100 delayed windows are opened providing high statistics accidental backgrounds). In addition to this selection a $100 \mu$ s muon-veto cut is applied and we require no events within $100 \mu$ s before and after an IBD candidate. The latter criteria suppress multineutron cascades created by cosmic radiation. Non-tagged muons that stop and decay in the top layer of the detector may be mistaken as IBD candidates. Their asymmetry of charge deposition in the PMTs of the vertex cell is higher than that of events in the detector bulk and was used to remove these stopping muon events. Note that muons stopping deeper inside the detector are rejected due to their high energy deposit before they stop. The segmentation of the detector is also used to reject IBD candidates with the wrong topology, by limiting the energy deposition of the prompt event to the vertex cell, only allowing for positron annihilation gammas in the other cells, and by a distance cut between prompt and delayed events.

\subsection{IBD-like background}

Whereas the contribution of random coincidences can be determined directly for each data set as described in 


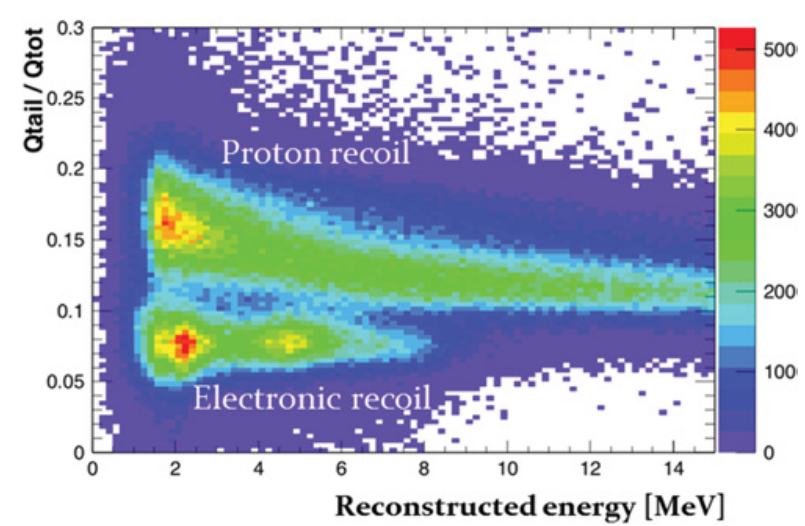

Figure 2. Distribution of the PSD value, defined as the ratio of the integrated charge in the tail of the pulse $\left(Q_{\text {tail }}\right)$ to the total charge $Q_{t o t}$, as a function of the reconstructed energy for the prompt signal of IBD-like events collected in cell 1 during reactor turned off period.

Sect. 3.1, coincidences between two physically correlated events can also pass the selection cuts and mimic IBD events. The origin of this correlated background is important to be known in order to correctly subtract it from the data to extract the anti-neutrino signal. Reactor turned off periods are used to measure this background. Up to now we have accumulated more than 138 days of reactor turned off data.

The distribution of PSD value for the prompt signal of those events is shown on Fig. 2 as a function of the reconstructed energy. Two distinct regions are populated: the proton recoil region due to energetic neutrons impinging on hydrogen atoms and the electron recoil region due to gamma-ray Compton scattering on electrons or lepton-electron scattering. The positron signal from IBD events populates the electron recoil region (see Fig. 2 in [4]).

The correlated background has thus two origins: energetic neutrons thermalized in the detector and then captured by gadolinium atoms (proton recoil region) and $\gamma-\gamma$ coincidences due to at least two neutrons inducing nuclear reactions in the coincidence window with gamma emissions (electron recoil region). At low energy, in the electron recoil region, very few events are also due to remaining stopping muons which are neither rejected by the muon veto, nor by the asymmetry of charge deposition in the PMTs. Thus correlated backgrounds mainly originate from cosmic radiation-induced neutrons which are produced directly in the atmosphere in a cosmic shower or more probably in the vicinity of the detector due to muon-induced reactions.

The shape of the background energy spectrum is important to be understood as structures could mimic oscillations in a shape analysis of the anti-neutrino rates. Fig. 3 shows background energy spectra of IBD-like events for the different contributions of proton and electron recoils and accidental coincidences. As observed and expected the accidental coincidences, dominated by decay gamma-rays, are below $3 \mathrm{MeV}$ reconstructed energy and the proton recoils induced by neutrons are more or less uniformely distributed in energy. A clear structure appears in the electronic recoil distribution with peaks attributed to $(n, \gamma)$ reactions on hydrogen at low energy $(2.2 \mathrm{MeV}$

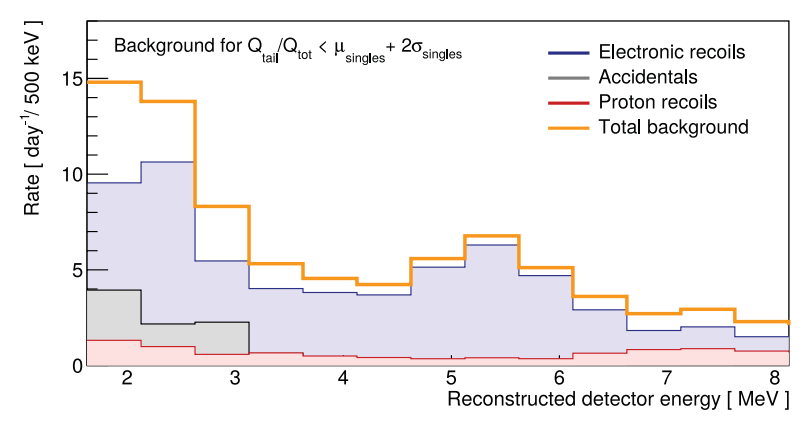

Figure 3. Background energy spectra for the different listed contributions in the IBD region obtained by selecting correlated events having PSD values less than 2 standard deviations from the mean value of the electronic recoil peak for single events. Data taken with reactor turned off.

gamma ray) and gadolinium at higher energy (broad peak above $8 \mathrm{MeV}$ in reconstructed energy). The peak around 5.5 MeV reconstructed energy is attributed to the $\left(n, n^{\prime} \gamma\right)$ reaction on ${ }^{12} \mathrm{C}$. In this reaction a $4.4 \mathrm{MeV}$ gamma-ray is emitted in the final state in coincidence with a neutron whose energy depends on the incident neutron energy and which is responsible for a broadening of the peak. This is confirmed by the good shape agreement when comparing with the energy spectra obtained with the AmBe source which produces a $4.4 \mathrm{MeV}$ gamma-ray in coincidence with a neutron of less than $10 \mathrm{MeV}$. The peak width indicates that the incident neutrons should have energies of a few $\mathrm{MeV}$ - considering a quenching factor of about 3 for the energy deposited by a neutron in the liquid - as expected for cosmic neutrons. We tested this hypothesis by simulating the neutron production by cosmic muons in the vicinity of the detector. From this simulation we observed that neutrons are mainly produced by spallation reactions in the lead shielding surrounding the detector, with energies distributed mainly around a few $\mathrm{MeV}$.

Whereas $(n, \gamma)$ reactions are pure electron recoil events, the $\left(\mathrm{n}, \mathrm{n}^{\prime} \gamma\right)$ reactions on ${ }^{12} \mathrm{C}$ mix electron recoil and proton recoil events. Therefore the resulting PSD distribution for those events should be slightly shifted compared to pure electromagnetic events. Choosing single events as the reference for pure electron recoil events we have measured the relative deviation of the PSD distribution for IBD-like background as a function of the energy. As can be seen in Fig. 4 the mean value of the PSD distribution of IBDlike background is located at higher values compared to the PSD distribution of pure electron recoil events above $5 \mathrm{MeV}$ with a maximum deviation of about $10 \%$. The same trend is observed for the width of the IBD-like PSD distribution. Note the small offset of about $2 \%$ between the two distributions which is probably due to a difference in the vertex position of the reactions.

\subsection{Neutrino rate extraction method}

A multi-Gaussian fit procedure of the PSD distributions is used to extract the anti-neutrino rates using reactor turned off and turned on data. In reference [4] the PSD distributions of correlated background measured with reactor turned off were fitted using a two Gaussians model to reproduce the electron- and proton-recoil regions. Thanks to the accumulation of turned off data in STEREO phase II we improved the fit by introducing a third 


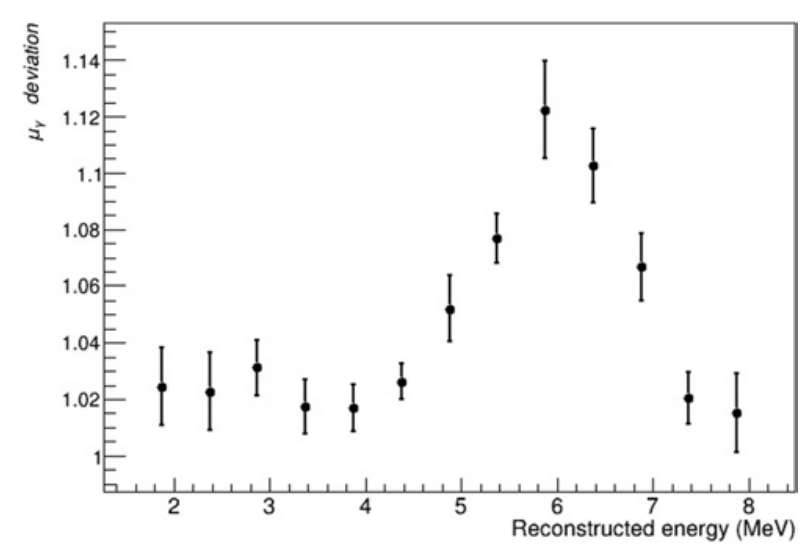

Figure 4. Relative deviation of the mean $\left(\mu_{\gamma}\right)$ electron-recoil PSD component for IBD-like background events compared to single events. Distributions were fitted with Gaussian functions using Eq. (2) for each cell and the average values over the six cells are reported. Uncertainties take into account the deviation between cells.

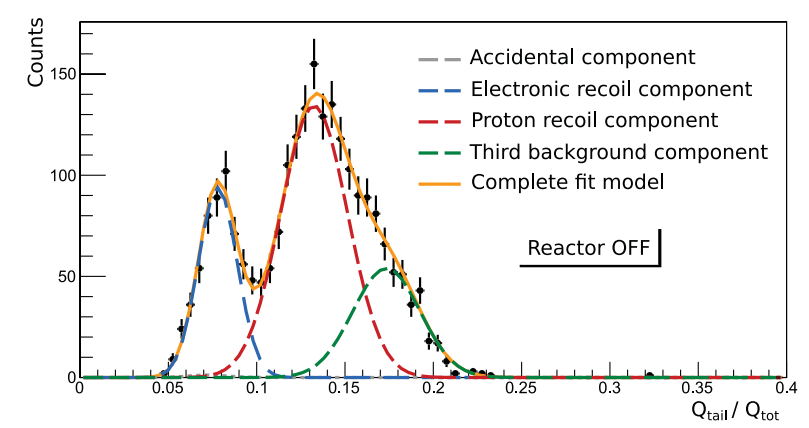

Figure 5. Distribution of the PSD value for events in cell 2 for reconstructed energy bin around $3.375 \mathrm{MeV}$, collected in 84 days of reactor turned off. The different components of the fit are also indicated, with the electronic component at low $Q_{\text {tail }} / Q_{\text {tot }}$ and the third Gaussian component at high $Q_{\text {tail }} / Q_{\text {tot }}$.

Gaussian to reproduce correctly the shoulder observed at high PSD values in the proton-recoil component (see Fig. 5). The background model then is written as:

$$
\begin{aligned}
M_{b c k}\left(q_{i}\right)= & \frac{A_{\gamma}}{A_{p}} G\left(q_{i}, \mu_{\gamma}, \sigma_{\gamma}\right)+\frac{A_{p 1}}{A_{p}} G\left(q_{i}, \mu_{p 1}, \sigma_{p 1}\right) \\
& +\frac{A_{p 2}}{A_{p}} G\left(q_{i}, \mu_{p 2}, \sigma_{p 2}\right)
\end{aligned}
$$

where $G(q, \mu, \sigma)$ is the normalized Gaussian distribution with $\mu$ and $\sigma$ the mean and standard deviation respectively, $A_{p}=A_{p 1}+A_{p 2}$ is the integral of the proton recoil component and $A_{\gamma} / A_{p}$ the ratio between the electron and proton components.

An example of a PSD distribution with 84 days of reactor turned off data fitted with the three Gaussian model is shown in Fig. 5. The contribution of accidental coincidences, dominated by $\gamma-\gamma$ coincidences, is added with a Gaussian distribution fitted to the data extracted with the delayed time windows. The model is written:

$$
\begin{aligned}
M^{O F F}\left(q_{i}\right)= & R_{a c c}^{O F F} \times G\left(q_{i}, \mu_{a c c}, \sigma_{a c c}\right) \\
& +R_{p}^{O F F} M_{b c k}^{O F F}\left(q_{i}\right)
\end{aligned}
$$

where $R_{p}^{O F F}$ is the correlated background rate and $R_{\text {acc }}^{O F F}$ the accidental rate measured separately.
The origin of the shoulder is not well established but it could originate from low energy multi-neutrons which induce multi-proton recoils each one having a low PSD value. The introduction of a third component improves the quality of the fit and provides a better description of the proton-recoil component contribution in the IBD-like region. For the phase I analysis [4] the model reduces to the two first Gaussians $\left(A_{p 2}=0\right)$. But as shown in Fig. 6 and coupled to another improvement detailed later, it does not impact the ratio between the anti-neutrino energy spectra measured in two cells and thus the results shown in the next section.

The same background model is used to fit the PSD distributions when the reactor is turned on with an additional Gaussian component to reproduce IBD events and is written:

$$
\begin{aligned}
M^{O N}\left(q_{i}\right)= & R_{v} \times G\left(q_{i}, \mu_{v}, \sigma_{v}\right)+R_{a c c}^{O N} \\
& \times G\left(q_{i}, \mu_{a c c}, \sigma_{a c c}\right)+R_{p}^{O N} M_{b c k}^{O N}\left(q_{i}\right)
\end{aligned}
$$

where $R_{v}$ is the anti-neutrino rate, $R_{p}^{O N}$ the correlated background rate and $R_{\text {acc }}^{O N}$ the accidental rate measured separately. $R_{\nu}, \mu_{\nu}, \sigma_{\nu}, R_{p}^{O N}, \mu_{p 1}$ and $\sigma_{p 1}$ are free parameters of the fit, $R_{p}^{O N}$ allowing to take into account the variations of the background as a function of time due to atmospheric pressure variations or changes of environmental conditions.

In Ref. [4], data were analyzed in time bins of 7 days to account for PSD time-variations and the fit procedure applies for each time bin and each energy bin. Low statistics in some bins required the use of a maximum likelihood based parameter estimation for all bins. For reactor turned on or turned off periods the global binned likelihood is written as:

$$
\mathcal{L}_{0}=\prod_{i} P\left(q_{i} \mid M\left(q_{i}\right)\right) \times P\left(q_{i}^{a c c} \mid M_{a c c}\left(q_{i}\right)\right)
$$

where $P()$ is the Poisson probability density function, $M\left(q_{i}\right)$ the model defined by Eq. (3) or Eq. (4) and $M_{a c c}\left(q_{i}\right)$ the model for accidental events.

In the fit procedures some of the parameters were constrained by adding Gaussian pull-terms to Eq. (5):

$$
\ln \left(\mathcal{L}^{O N}\right)=\ln \left(\mathcal{L}_{0}^{O N}\right)+\sum_{\alpha} \frac{\left(\mu_{\alpha}-\left\langle\mu_{\alpha}\right\rangle\right)^{2}}{2 \sigma_{\alpha}^{2}}
$$

where $\mu_{\alpha}$ is the parameter and $\left\langle\mu_{\alpha}\right\rangle$ and $\sigma_{\alpha}$ are the mean and standard deviation of the Gaussian distribution in which the parameter is allowed to take values. In the following we describe which parameters are constrained.

In Ref. [4], the PSD distributions of single events were used as reference to determine the Gaussian parameters of the electron-recoil components. In the fit procedures, the means and standard deviations of the electron-recoil Gaussians were fixed to the values of the single event Gaussian $\left(\mu_{\gamma}=\mu_{a c c}=\mu_{s}, \sigma_{\gamma}=\sigma_{a c c}=\sigma_{s}\right)$. In a recent improvement of the method we include the average deviations observed on Fig. 4 with their uncertainties to correct single data parameters. This correction is important to apply as it affects the anti-neutrino energy spectrum shape above $5 \mathrm{MeV}$ and creates a small bias in the rate at lower energy. But as shown on Fig. 6, coupled to the previous improvement on the background model, it does 


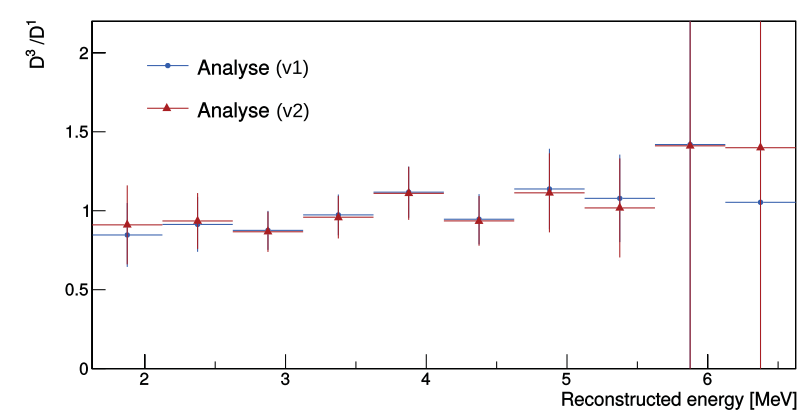

Figure 6. Comparison of the IBD energy spectrum ratios between cell 3 and cell 1 when using single events as reference for the fit of the electronic component (v1) or the deviation from single events (v2) (see text). The IBD energy spectrum in v1 analysis was obtained with a two-Gaussians model to fit the IBD-like background whereas the three-Gaussians model is used in v2 analysis.

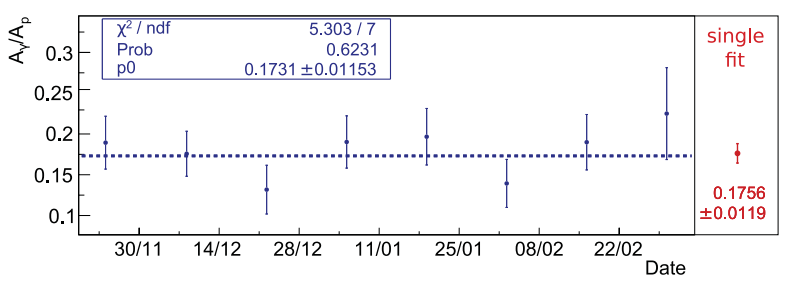

Figure 7. $A_{\gamma} / A_{p}$ as a function of time for reactor turned off period and for one energy bin [2.675-3.125 MeV] in cell 4. Blue symbols are 14 days of data and the red symbol is integrated data over the whole reactor turned off period.

not affect the ratio of anti-neutrino energy spectra of two cells and preserves results from [4].

For reactor turned on periods the background electronrecoil to proton-recoil populations ratio $\left(A_{\gamma}^{O N} / A_{p}^{O N}\right)$ was constrained by reactor-off data assuming no dependence on time. The background studies have shown that these two populations originate from the same physical processes and thus should follow the same variations due atmospheric pressure or environmental changes. Figure 7 presents this ratio extracted from the fit procedure on reactor turned off data for time bins and for an integrated bin over the whole reactor turned off period. No dependence with time is observed, at least within the uncertainties. The mean value extracted from the fit of the ratios over time bins is in very good agreement with the value obtained when integrating over the whole period with more statistics. This shows that no significant bias is introduced by the fit procedure. For each energy bin and cell, a mean $A_{\gamma}^{O F F} / A_{p}^{O F F}$ value was extracted by this way with its uncertainty and used to constrain the equivalent ratio $A_{\gamma}^{O N} / A_{p}^{O N}$ for reactor turned on periods.

The anti-neutrino parameters were also slightly constrained taking into account that the PSD distribution of IBD prompt events is slightly shifted compared to pure gamma-ray PSD. This was observed by subtracting reactor turned on and reactor turned off distributions and could be explained by the fact that the energy deposition is slightly different between positron and gamma-ray. Such deviation is accounted for by constraining moderately the mean and the width of the IBD Gaussian with respect to singles: $\mu_{v}=\mu_{s} \pm 0.2 \sigma_{s}$ and $\sigma_{v}=(0.95 \pm 0.10) \sigma_{s}$.

Finally, the anti-neutrino rate for each energy bin results from a fit on extracted anti-neutrino rates

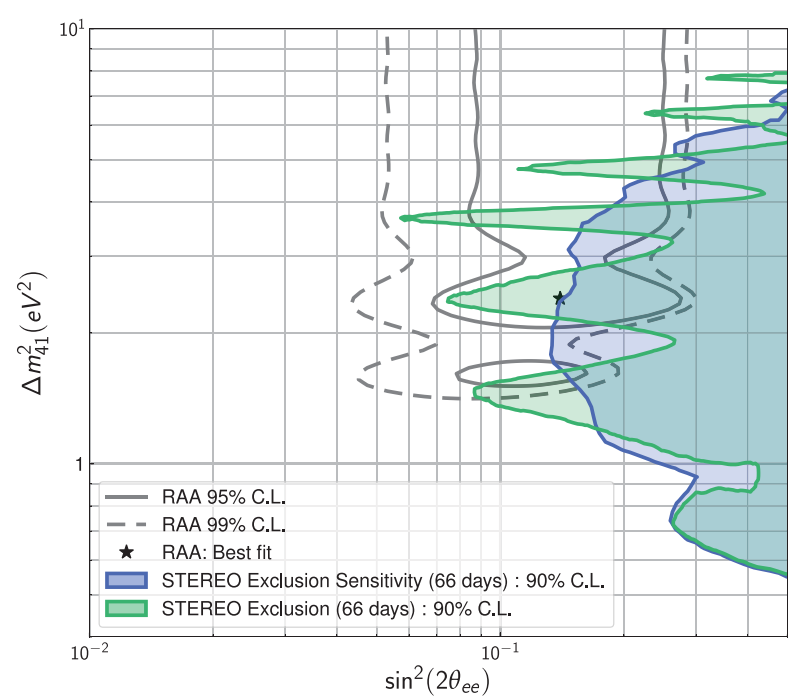

Figure 8. Exclusion contour plot of the oscillation parameter space (from [4]).

normalized to the reactor power for each time bin with a constant function. An asymmetric Chi-square function is used for the minimization procedure. It results in a total anti-neutrino rate of $(396.3 \pm 4.7) \bar{v}_{e}$ /day with a signal to background ratio of about 0.9 .

\section{Results and conclusions}

The IBD rate ratio between two cells was used to test for a possible oscillation toward a sterile neutrino in the data. The spectra of cells 2 to 6 are divided, bin by bin, by the spectrum of cell 1 , which serves as reference, and the ratios obtained with the data are compared to the ratios obtained from a Monte-Carlo simulation. This method is insensitive to the spectrum prediction and exploits the relative difference between the cells, thus getting rid of common systematics. The Monte-Carlo simulation of the detector is based on Geant4 libraries and includes all the detector responses allowing some systematic uncertainties to be estimated. A profile $\Delta \chi^{2}$ method was used with

$$
\begin{aligned}
\chi^{2}= & \sum_{i=1}^{N_{\text {ebin }}}\left(\overrightarrow{R_{i}^{\text {data }}}-\overrightarrow{R_{i}^{M C}}(\alpha)\right)^{t} V_{i}^{-1}\left(\overrightarrow{R_{i}^{\text {data }}}-\overrightarrow{R_{i}^{M C}}(\alpha)\right) \\
& +\sum_{j=1}^{N_{\text {cells }}}\left(\frac{\alpha_{j}^{\text {norm }}}{\sigma_{j}^{\text {norm }}}\right)^{2}+\sum_{j=1}^{N_{\text {cells }}}\left(\frac{\alpha_{j}^{\text {Escale }}}{\sigma_{j}^{\text {Escale }}}\right)^{2}
\end{aligned}
$$

where $\overrightarrow{R_{i}^{\text {data }}}$ and $\overrightarrow{R_{i}^{M C}}(\alpha)$ are five-dimensional vectors for the measured and the MC ratios, for the $i^{\text {th }}$ energy bin. The correlations between cells are coded into the covariance matrix and determined by random sampling assuming normal distributions for the anti-neutrino rate uncertainties. The correlated uncertainties are treated as nuisance parameters: $\alpha_{j}^{\text {norm }}$ are the relative normalizations $\left(\sigma_{l \neq 4}^{\text {norm }}=1.7 \%\right.$ and $\sigma_{4}^{\text {norm }}=3.4 \%$ in Phase- I $), \alpha_{j>0}^{\text {Escale }}$ the energy scale discrepancies between cells $\left(\sigma_{j>0}^{\text {Escale }}=\right.$ $1.1 \%)$, and $\alpha_{j=0}^{\text {Escale }}$ the common energy scale $\left(\sigma_{j=0}^{\text {Escale }}=\right.$ $0.35 \%$ ). The analysis was limited to energies below $7.125 \mathrm{MeV}$ to avoid denominators close to zero within a few $\sigma$ units in the ratios.

Results with 66 days of reactor turned on data for the different ratios are presented in Fig. 3 of Ref. [4]. We report 
here only the conclusion that data are compatible with the null oscillation hypothesis which cannot be rejected. A raster scan method was used to produce the exclusion contour plot at $90 \%$ C.L. It is shown on Fig. 8 together with the sensitivity of the experiment leading to the conclusion that oscillations with amplitude greater than $15-20 \%$ can be excluded at more than $90 \%$ C.L in the mass squared difference range of $1-5 \mathrm{eV}^{2}$. This conclusion is in agreement with results from other anti-neutrino reactor experiments DANSS [7] and PROSPECT [8].

These results demonstrate the ability of the STEREO experiment to detect anti-neutrinos above the residual background partly rejected thanks to the passive shieldings and the PSD features of the liquid. In this paper we have identified the origin of the residual background as nuclear reactions induced by cosmic-radiation produced neutrons in the liquid scintillator. This led us to improve the rejection methodology of the background and to reduce the bias on the anti-neutrino energy spectra. Even if structured in energy, the shape of the background is expected to be stable in time. This conclusion with the methodology improvements are important results for the next step of the STEREO experiment which is to provide the anti-neutrino energy spectrum associated to the fission of ${ }^{235} \mathrm{U}$ and to test if this fissile isotope is responsible for the shape distortion observed above 4-5 MeV.
This work is supported by the French National Research Agency (ANR) within the project No. ANR-13-BS05-0007 and the programs P2IO LabEx (ANR-10-LABX-0038) and ENIGMASS LabEx (ANR-11-LABX-0012). We acknowledge the support of the CEA, CNRS/IN2P3, the ILL and the Max-PlanckGesellschaft.

\section{References}

[1] T.A. Mueller et al., Phys. Rev. C 83, 054615 (2011)

[2] P. Huber, Phys. Rev. C 84, 024617 (2011)

[3] G. Mention et al., Phys. Rev. D 83, 073006 (2011)

[4] H. Almazan et al. (STEREO collaboration), Phys. Rev. Lett. 121, 161801 (2018)

[5] C. Buck, A.P. Collin, J. Haser, M. Lindner, Phys. Lett. B 765, 159 (2017); G. Mention et al., Phys. Lett. B 773, 307 (2017)

[6] N. Allemandou et al. (STEREO collaboration), J. Instrum. 13, P07009 (2018)

[7] I. Alekseev et al. (DANSS collaboration), Phys. Lett. B 787, 56 (2018)

[8] J. Ashenfelter et al. (PROSPECT collaboration), Phys. Rev. Lett. 121, 251802 (2018) 